\title{
Alterações hepáticas em codornas japonesas submetidas à intoxicação prolongada por aflatoxina $B_{1}$
}

\author{
Hepatic changes in japanese quail after long term intoxication by aflatoxin $B_{1}$
}

\author{
Carlos Augusto Fernandes Oliveira ${ }^{1}$ Paula Butkeraitis ${ }^{2}$ Josué Fontes Rosmaninho ${ }^{3}$ \\ José Luiz Guerra ${ }^{4}$ Benedito Correa $^{5}$ Tatiana Alves Reis ${ }^{6}$
}

\section{RESUMO}

\begin{abstract}
$O$ objetivo do presente trabalho foi estudar os efeitos da aflatoxina $B_{1}(A F B$ ) sobre as vísceras (fígado, baço e moela) de codornas poedeiras japonesas, em condições de exposição a baixas doses, tendo em vista que são poucos os dados de toxicidade de longa duração nesta espécie. Assim, foram constituídos 4 grupos formados, cada um, por 6 codornas de linhagem comercial, as quais receberam rações contendo AFBı nas concentrações de 0 (controle), 25, 50 e $100 \mu \mathrm{g} \mathrm{kg}^{-1}$, durante 168 dias. As aves do grupo $100 \mu \mathrm{g} \mathrm{kg}^{-1}$ apresentaram fígados com peso relativo médio menor $(p<0,05)$ do que o controle. Alterações histológicas foram constatadas apenas no fígado, sendo que todas as amostras provenientes das codornas intoxicadas apresentaram degeneração vacuolar macrogoticular, sugestivas de metamorfose gordurosa moderada a severa, particularmente nos grupos que receberam ração com os maiores níveis de $A F B_{1}\left(50\right.$ e $\left.100 \mu \mathrm{g} \mathrm{kg}^{-1}\right)$. A hiperplasia de ductos biliares foi observada somente no grupo alimentado com $100 \mu \mathrm{g}^{-1}$. Os resultados indicaram que a $A F B_{1}$, a partir de $50 \mu \mathrm{g} \mathrm{kg}^{-1}$, pode ocasionar lesões hepáticas significativas em codornas de postura, em condições de exposição prolongada.
\end{abstract}

Palavras-chave: aflatoxina $B$, toxicidade, alterações histopatológicas, codornas.

\section{ABSTRACT}

The aim of the present record was to study the effects of aflatoxin $B_{1}\left(A F B_{1}\right)$ on selected viscera (liver, spleen and gizzard) of laying Japanese quail under conditions of low level exposure, in view of the little information regarding the long term toxicity on this specie. Thus, four experimental groups of six commercial quails were constituted and given rations containing either 0 (controls), 25, 50 or $100 \mu \mathrm{g}$ aflatoxin $\mathrm{B}_{1}$ $\left(A F B_{1}\right) / k g$ feed, during 168 days. When compared to controls, birds from group $100 \mu \mathrm{g} / \mathrm{kg}$ presented low relative liver weight ( $p<0.05)$. Histological changes were observed only in the livers, and all samples from quail exposed to AFBI revealed moderate to severe hepatic cell vacuolation with fatty change, particularly in birds from groups receiving highest levels of toxin $(50$ and $100 \mu \mathrm{g} / \mathrm{kg})$. Bile duct hyperplasia occurred only in the birds exposed to $100 \mu \mathrm{g} / \mathrm{kg}$ of $A F B$. The results indicated that long term administration of $A F B_{1}$ at levels above $50 \mu \mathrm{g} / \mathrm{kg}$ can cause significant hepatic lesions in Japanese quail.

Key words: aflatoxin B1, toxicity, histopathology, quail.

\section{INTRODUÇÃO}

As aflatoxinas são metabólitos hepatotóxicos produzidos por fungos do gênero Aspergillus, espécies A.flavus, A.parasiticus e A.nomius (MOSS, 1998). Os principais produtos alimentícios susceptíveis ao desenvolvimento destes fungos incluem milho e trigo que, normalmente, podem ser utilizados na composição de rações empregadas em avicultura (LEESON et al., 1995).

São conhecidos, atualmente, 18 compostos similares designados pelo termo aflatoxina, porém os principais tipos de interesse médico-sanitário são identificados como $B_{1}, B_{2}, G_{1}$ e $G_{2}$ (COULOMBE, 1991).

${ }^{1}$ Médico Veterinário, Professor Associado da Faculdade de Zootecnia e Engenharia de Alimentos da Universidade de São Paulo (USP), Av. Duque de Caxias Norte 225, 13630-000, Pirassununga, SP, Brasil. E-mail: carlosaf@usp.br Autor para correspondência.

${ }^{2}$ Médico Veterinário, Aluno de Pós-graduação da Faculdade de Medicina Veterinária e Zootecnia (FMVZ), USP.

${ }^{3}$ Médico Veterinário, Aluno de Pós-graduação da Faculdade de Saúde Pública, USP.

${ }^{4}$ Médico Veterinário, Professor Doutor da FMVZ, USP.

${ }^{5}$ Biomédico, Professor Associado do Instituto de Ciências Biomédicas (ICB), USP.

${ }^{6}$ Médico Veterinário, Técnico Especializado de Nível Superior do ICB,USP. 
A estrutura química destes compostos é muito semelhante, sendo que todas apresentam um núcleo central cumarínico ligado a uma estrutura bi-furanóide. As aflatoxinas, no entanto, apresentam diferentes graus de atividade biológica: a aflatoxina $\mathrm{B}_{1}\left(\mathrm{AFB}_{1}\right)$, além de ser a mais freqüentemente encontrada em substratos vegetais, é a que apresenta maior poder toxigênico, enquanto que a toxicidade aguda das aflatoxinas $\mathrm{G}_{1}, \mathrm{~B}_{2}$ e $\mathrm{G}_{2}$ é cerca de 50,20 e $10 \%$ da $\mathrm{AFB}_{1}$, respectivamente (LEESON et al., 1995).

A ocorrência de aflatoxinas, no Brasil, tem sido observada com freqüência, principalmente no Estado de São Paulo, em alimentos destinados ao consumo humano e animal. Os resultados de alguns levantamentos sobre os níveis de aflatoxinas encontrados em ingredientes para rações e rações prontas indicam um elevado percentual de amostras positivas, principalmente em rações prontas, sendo descritos valores de 10,4 a 56,9\% para estes produtos (SABINO, 1980; SABINO et al., 1988; SANTURIO et al., 1992; RIBEIRO et al., 2000). As concentrações médias de aflatoxinas no milho e em rações são muito variáveis, porém, foram reportados níveis de até $7.800 \mu \mathrm{g} \mathrm{kg}^{-1}$ (SABINO, 1980), portanto, potencialmente capazes de originar efeitos adversos nas espécies de exploração avícola.

Os efeitos tóxicos das aflatoxinas são dependentes da dose e do tempo de exposição, determinando, assim, intoxicações agudas ou crônicas. A síndrome tóxica aguda ocorre pela ingestão de alimento com alta concentração de aflatoxina, sendo os efeitos observados em curto espaço de tempo. Caracteriza-se principalmente pela rápida deterioração do estado geral do animal, perda de apetite, hepatite aguda, icterícia, hemorragias e morte (LEESON et al., 1995). O fígado é o principal órgão afetado, com lesões decorrentes da necrose hemorrágica, proliferação das células dos ductos biliares e infiltração gordurosa dos hepatócitos (ESPADA et al., 1992; OLIVEIRA et al., 1999).

A sensibilidade aos efeitos tóxicos das aflatoxinas varia consideravelmente entre as espécies animais. Com relação às espécies exploradas na avicultura comercial, a susceptibilidade é maior em patos, seguidos de perus, gansos, faisões e frangos (MULLER et al., 1970). Mesmo entre indivíduos de uma mesma espécie, a relação dose-resposta pode variar de acordo com raça, sexo, idade e composição da dieta, entre outros fatores (COULOMBE, 1991).

$\mathrm{Na}$ aflatoxicose crônica, o sinal clínico mais evidente é a diminuição da taxa de crescimento dos animais jovens (LEESON et al., 1995). A doença originase, de modo geral, a partir da ingestão de alimentos contaminados com baixos níveis de aflatoxinas por um longo período de tempo, constituindo a principal forma de intoxicação em condições naturais, o que ocasiona perdas econômicas consideráveis às criações animais (PIER, 1992).

As codornas apresentam elevada sensibilidade às aflatoxinas, conforme se observa pelos resultados de alguns estudos de toxicidade aguda efetuados em codornas de postura (SAWHNEY et al., 1973; CHANG \& HAMILTON, 1982). Contudo, os estudos sobre os efeitos de baixas concentrações de aflatoxinas em codornas são escassos na literatura, existindo apenas dois trabalhos conhecidos. Em um deles, JOHRI et al. (1990) empregaram níveis de 200 a $750 \mu \mathrm{g}$ de aflatoxinas (sem especificar quais frações) por kg de ração durante 100 dias, observando fígados pálidos e com áreas necrosadas nas aves de todos os grupos, além de diminuição do peso corpóreo das aves dos tratamentos acima de $500 \mu \mathrm{g} \mathrm{kg}^{-1}$. BINTVIHOK et al. (1993), por outro lado, alimentaram codornas com rações contendo 50, 100 e $200 \mu \mathrm{g}$ de $\mathrm{AFB}_{1} / \mathrm{kg}$ durante 84 dias, e observaram somente hiperplasia moderada de ductos biliares em 3 aves do tratamento $200 \mu \mathrm{g} \mathrm{kg}^{-1}$, e 1 do tratamento $100 \mu \mathrm{g} \mathrm{kg}^{-1}$.

O objetivo do presente trabalho foi verificar os efeitos da $\mathrm{AFB}_{1}$ sobre vísceras (fígado, baço e moela) de codornas poedeiras alimentadas com rações contendo baixas concentrações da toxina por período prolongado.

\section{MATERIAL E MÉTODOS}

Foram utilizadas 24 codornas de linhagem Japonesa (Coturnix coturnix japonica) adquiridas com 5 semanas de idade e já vacinadas, subdivididas em 4 grupos experimentais de 6 aves. As aves foram mantidas em adaptação por 2 semanas, sendo alimentadas com ração convencional à base de milho e farelo de soja, isenta de $\mathrm{AFB}_{1}$, formulada de modo a atender às exigências nutricionais de aves em postura comercial, conforme as recomendações do NATIONAL RESEARCH COUNCIL (1994). As aves foram alojadas em bateria contendo 4 gaiolas de arame providas de comedouros lineares e bebedouros tipo calha em "V" para água corrente.

$\mathrm{A} \mathrm{AFB}_{1}$ utilizada na intoxicação dos animais foi produzida a partir do cultivo de cepa toxigênica de A. flavus (IMI 190) $70 \%$ de pureza, proveniente do "International Micological Institute", Londres. Pequenos fragmentos da colônia de $\boldsymbol{A}$. flavus, mantida em ágar Czapek a $25^{\circ} \mathrm{C}$, foram inoculados no centro de placas de Petri contendo Agar Coco (LIN \& DIANESE, 1976), após o que foram incubadas a $25^{\circ} \mathrm{C}$ por 10 dias. 
Os cultivos foram submetidos à extração com clorofórmio, sendo o filtrado obtido evaporado a $60^{\circ} \mathrm{C}$. A determinação da concentração de $\mathrm{AFB}_{1}$ no extrato final foi realizada através de cromatografia de camada delgada (CCD), de acordo com SCOTT (1990).

O extrato final foi ressuspendido em óleo de milho previamente submetido à análise de aflatoxinas, com a finalidade de facilitar o preparo das rações contaminadas (ALMEIDA et al., 1996). Adotou-se a proporção total de $0,5 \%$ (v/p) de óleo de milho em relação à quantidade de ração preparada, com a finalidade de evitar, ao máximo, o desbalanceamento dos níveis energéticos das rações. Deste modo, as soluções contendo $\mathrm{AFB}_{1}$ foram diluídas em volumes convenientes de óleo de milho, de maneira a obter, na ração final, níveis de 0 (controle, apenas com óleo de milho), 25, 50 e 100 $\mu \mathrm{g} \mathrm{kg}^{-1}$ de AFB . A adição das soluções de toxina às rações foi reallizada em um misturador horizontal/helicoidal (Marconi ${ }^{\circledR}$ ), sendo a confirmação dos níveis da toxina adicionada efetuada por CCD, de acordo com SOARES \& RODRIGUEZAMAYA(1989).

O período de intoxicação foi iniciado quando as aves alcançaram 7 semanas de idade. $O$ estudo teve a duração total de 168 dias, durante os quais as aves receberam diariamente cerca de $30 \mathrm{~g}$ ração ave ${ }^{-1}$ e água ad libitum. Ao final do experimento, as aves foram pesadas individualmente e anestesiadas com éter etílico. Em seguida, foram sacrificadas por deslocamento cervical e imediatamente necropsiadas, sendo as vísceras pesadas e examinadas macroscopicamente para observação de possíveis lesões. Fragmentos retirados do fígado, baço e moela foram recolhidos em frascos contendo formalina a $10 \%$ tamponada, e encaminhados para exame histopatológico (VASCONCELOS, 1988). No laboratório, secções representativas dos tecidos (espessura de $5 \mu \mathrm{m}$ ) foram coradas com hematoxilinaeosina, sendo posteriormente submetidas ao exame em microscópio de luz. Os pesos absolutos das vísceras

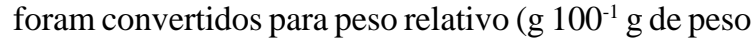
corpóreo - p.c.) e submetidos à análise de variância (SAS, 1992), utilizando-se, para a comparação entre as médias, o teste de Tukey $(\mathrm{p}<0,05)$.

\section{RESULTADOS E DISCUSSÃO}

No decorrer do experimento, não foram detectadas modificações visíveis no estado geral das aves, com exceção de 4 que vieram a óbito, sendo 1 do grupo alimentado com ração contendo $25 \mu \mathrm{g} \mathrm{kg}^{-1}$ (morta no $124^{\circ}$ dia do experimento), 1 do grupo $50 \mu \mathrm{g} \mathrm{kg}^{-1}$ (morta no $128^{\circ}$ dia do experimento) e 2 do grupo que recebeu $100 \mu \mathrm{g} \mathrm{kg}^{-1}$ (mortas no $24^{\circ}$ e $69^{\circ}$ dia do experimento). As vísceras (fígado, moela e baço) destas aves não apresentaram alterações visíveis após a necropsia, sendo que apenas o fígado de uma das aves do tratamento $100 \mu \mathrm{g} \mathrm{kg}^{-1}$ mostrou, ao exame histopatológico, áreas focais de necrose associada a processo inflamatório. Estes sinais concordam com os resultados obtidos por JOHRI et al. (1990), para níveis de aflatoxinas acima de $500 \mu \mathrm{g} \mathrm{kg}^{-1}$.

Não foram constatadas alterações macroscópicas significativas nas vísceras provenientes dos animais sacrificados ao final do experimento, com exceção de 2 amostras de fígado (grupos 50 e $100 \mu \mathrm{g} \mathrm{kg}^{-1}$ ), as quais se apresentaram com coloração amarelada.

A tabela 1 apresenta o peso corporal das aves imediatamente após o sacrifício, bem como os pesos relativos do baço, moela e fígado. Não houve diferenças significativas $(\mathrm{p}>0,05)$ no peso das aves, cujos valores estiveram entre 133,80 a $144,20 \mathrm{~g}$. Os valores médios obtidos para o baço foram semelhantes em todos os tratamentos $\left(0,04\right.$ a $0,05 \mathrm{~g} 100^{-1}$ g p.c. $)$. As médias de peso relativo da moela, por outro lado, foram superiores nos grupos intoxicados $\left(2,53\right.$ a 2,68g $100^{-1}$ g p.c.), porém, as diferenças com o valor obtido no grupo controle $\left(2,29 \mathrm{~g} 100^{-1} \mathrm{~g}\right.$ p.c.) não foram

Tabela 1 - Peso corpóreo e pesos relativos de vísceras de codornas japonesas, de acordo com a concentração de $\mathrm{AFB}_{1}$ na ração fornecida às aves $^{1}$.

\begin{tabular}{|c|c|c|c|c|}
\hline \multirow{2}{*}{$\mathrm{AFB}_{1}$ na ração $(\mu \mathrm{g} / \mathrm{kg})$} & \multirow{2}{*}{ Peso corpóreo (g) } & \multicolumn{3}{|c|}{ Pesos relativos (g/100 g de peso corpóreo) } \\
\hline & & Baço & Moela & Fígado \\
\hline 0 (controle) & $144,20 \pm 7,54^{\mathrm{a}}$ & $0,04 \pm 0,01^{\mathrm{a}}$ & $2,29 \pm 0,31^{a}$ & $3,80 \pm 0,57^{\mathrm{a}}$ \\
\hline 25 & $133,80 \pm 5,79^{a}$ & $0,04 \pm 0,01^{\mathrm{a}}$ & $2,67 \pm 0,25^{\mathrm{a}}$ & $3,49 \pm 0,66^{\mathrm{a}}$ \\
\hline 50 & $136,95 \pm 7,76^{\mathrm{a}}$ & $0,04 \pm 0,02^{a}$ & $2,68 \pm 0,42^{a}$ & $3,52 \pm 0,34^{\mathrm{a}}$ \\
\hline 100 & $139,27 \pm 5,62^{\mathrm{a}}$ & $0,05 \pm 0,01^{\mathrm{a}}$ & $2,53 \pm 0,26^{a}$ & $2,96 \pm 0,32^{b}$ \\
\hline
\end{tabular}

${ }^{\mathrm{a}, \mathrm{b}}$ Em uma coluna, médias seguidas de letras iguais não diferem estatisticamente $(\mathrm{p}>0,05)$.

${ }^{1}$ Valores se referem à média \pm desvio padrão para 6 aves em cada tratamento. 
significativas ( $\mathrm{p}>0,05)$. Estes resultados são similares aos dados obtidos por CHANG \& HAMILTON (1982), os quais não observaram diferenças nos pesos relativos do baço e moela em codornas japonesas alimentadas com ração contendo níveis de 1.250 a $20.000 \mu \mathrm{g} \mathrm{kg}^{-1} \mathrm{de}$ aflatoxinas (das quais $70 \%$ era composta por $\mathrm{AFB}_{1}$ ), durante 4 semanas.

Os pesos relativos dos fígados das aves que receberam ração com $\mathrm{AFB}_{1}$ foram menores $(2,96 \mathrm{a}$ $3,49 \mathrm{~g} 100^{-1} \mathrm{~g}$ p.c.) em relação ao controle $\left(3,80 \mathrm{~g} 100^{-1} \mathrm{~g}\right.$ p.c.), sendo a diferença significativa $(\mathrm{p}<0,05)$ somente para o grupo $100 \mu \mathrm{g} / \mathrm{kg}$. Inversamente, CHANG \& HAMILTON (1982) observaram aumento do peso relativo do fígado de codornas, bem como uma redução de até $40 \%$ no peso corpóreo das aves, à medida que o nível de aflatoxinas na ração aumentou de 1.250 até $20.000 \mu \mathrm{g} \mathrm{kg}^{-1}$. Deve-se ressaltar que os níveis de aflatoxinas utilizados pelos autores foram extremamente elevados, ou seja, 12,5 a 200 vezes acima do nível máximo utilizado no presente trabalho, de modo que o resultado obtido por CHANG \& HAMILTON (1982) pode ser atribuído, em grande parte, à diminuição drástica do peso corpóreo das aves provocado pelos níveis elevados de aflatoxinas. Em nosso experimento, o peso corpóreo das aves não apresentou diferenças significativas entre os tratamentos, o que permite considerar que a diminuição do peso relativo do fígado das aves do tratamento $100 \mu \mathrm{g} \mathrm{kg}^{-1}$ foi devida, exclusivamente, aos efeitos diretos da $\mathrm{AFB}_{1}$ sobre este órgão, similarmente ao que ocorre com outras espécies avícolas, em condições de exposição a baixas concentrações na ração (LEESON et al., 1995).

As amostras de baço e moela, coletados das aves dos grupos controle, 25,50 e $100 \mu \mathrm{g} \mathrm{kg}^{-1}$ não apresentaram alterações histopatológicas significativas. Com relação ao fígado, as amostras provenientes do grupo controle também não apresentaram lesões aparentes, porém, algumas áreas isoladas apresentaram-se com discreta degeneração vacuolar do tecido hepático. Este fato pode estar relacionado, provavelmente, à esteatose fisiológica observada em praticamente todas as aves em postura, em condições naturais (RANDALL \& REECE, 1996).

Os fígados das aves que receberam rações contendo 25 e $50 \mu \mathrm{g} \mathrm{kg}^{-1}$ de $\mathrm{AFB}_{1}$ apresentaram somente áreas restritas de esteatose, em menor grau do que o grupo alimentado com $100 \mu \mathrm{g} \mathrm{kg}^{-1}$, não sendo observadas diferenças entre ambos os grupos. Algumas amostras do grupo $50 \mu \mathrm{g} / \mathrm{kg}$ apresentaram-se mais intensamente vacuolizadas, indicando uma possível relação dose-dependente para este efeito. Os dados apresentados discordam dos resultados obtidos por BINTVIHOK et al. (1993), os quais não encontraram sinais de degeneração gordurosa em codornas alimentadas com $\mathrm{AFB}_{1}$ nos níveis de 50 a $200 \mu \mathrm{g} \mathrm{kg}^{-1}$, durante 84 dias. As diferenças entre ambos os estudos podem estar associadas a diversos fatores, principalmente o período de intoxicação e as variações na susceptibilidade de diferentes linhagens de codornas à $\mathrm{AFB}_{1}$ (LEESON et al., 1995).

Todas as amostras de fígado do grupo $100 \mu \mathrm{g}$ $\mathrm{kg}^{-1}$ revelaram intensa degeneração vacuolar macrogoticular, bem como alteração da estrutura geral dos hepatócitos, conforme pode ser observado na figura 1. Algumas amostras exibiram áreas pronunciadas de hiperplasia de ductos biliares e desarranjo trabecular, sendo que estas alterações podem ser consideradas características dos efeitos da $\mathrm{AFB}_{1}$ no fígado (ESPADA et al., 1992), tendo sido reportada, também, em galinhas poedeiras alimentadas por períodos prolongados com ração contendo $\mathrm{AFB}_{1}$ a partir de $300 \mu \mathrm{g} \mathrm{kg}^{-1}$ (OLIVEIRA et al., 1999).

\section{CONCLUSÕES}

A administração prolongada de $100 \mu \mathrm{g} / \mathrm{kg}$ de $\mathrm{AFB}_{1}$ na ração de codornas diminuiu significativamente o peso relativo do fígado das aves. Adicionalmente, níveis de 50 e $100 \mu \mathrm{g} \mathrm{kg}^{-1}$ de $\mathrm{AFB}_{1}$ ocasionaram lesões hepáticas caracterizadas por degeneração vacuolar macrogoticular, desarranjo trabecular e hiperplasia de células de ductos biliares, que constituem um importante indicador de toxicidade crônica nesta espécie.

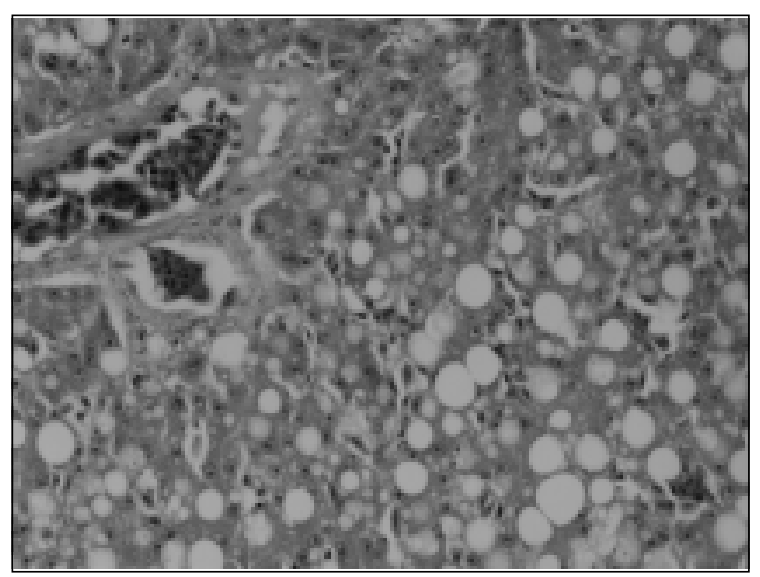

Figura 1 - Fotomicrografia de corte histológico de fígado de codorna do grupo $100 \mu \mathrm{g} / \mathrm{kg}$, evidenciando o comprometimento hepatocitário na zona 2 do ácino de Rapapport. Coloração: Hematoxilina-eosina. Aumento: 140x. 


\section{REFERÊNCIASBIBLIOGRÁFICAS}

ALMEIDA, R.M.A. et al. Acute effect of aflatoxin B on different inbred mouse strains II. Mycopathologia, v.133, p.2329, 1996.

BINTVIHOK, A. et al. Toxic effects of dietary aflatoxin and its residue in tissues and eggs in laying quails. In: INTERNATIONAL SYMPOSIUM OF THE WORLD ASSOCIATION OF VETERINARY FOOD HYGIENISTS, 1993, Bangkok. Proceedings... Bangkok : World Association of Veterinary Food Hygienists, 1993. p.299-307.

CHANG, C.F.; HAMILTON, P.B. Experimental aflatoxicosis in young japanese quail. Poult Sci, v.61, p.869-874, 1982.

COULOMBE, R.A. Aflatoxins. In: SHARMA, R.P.; SALUNKHE, D.K. Mycotoxins and phytoalexins. Boca Raton: CRC, 1991. p.103-143.

ESPADA, Y. et al. Pathological lesions following an experimental intoxication with aflatoxin $\mathrm{B}_{1}$ in broiler chickens. Res Vet Sci, v.53, p.275-279, 1992.

JOHRI, T.S.; AGRAWAL, R.; SADAGOPAN, V.R. Effect of low dietary levels of aflatoxin on laying quails (Coturnix coturnix japonica) and their response to dietary modifications. Indian J Anim Sci, v.60, p.355-359, 1990

LEESON, S.; DIAZ, G.J.; SUMMERS, J.D. Poultry metabolic disorders and mycotoxins. Guelph : University Books, 1995. $352 \mathrm{p}$.

LIN, M.T.; DIANESE, J.C. A coconut agar medium for rapid detection of aflatoxin production by Aspergillus spp. Phytopathology, v.66, p.1466-1469, 1976.

MOSS, M.O. Recent studies of mycotoxins. J Applied Microbiol Symposium, v.84, p.62S-76S, 1998.

MULLER, R.D. et al. The response of chicks, ducklings, goslings, pheasants and poults to graded levels of aflatoxin Poult Sci, v.49, p.1346-1350, 1970.

NATIONAL RESEARCH COUNCIL. Nutrient requirements of poultry. 9.ed. Washington : National Academy of Sciences, 1994. $155 \mathrm{p}$

OLIVEIRA, C.A.F. et al. Hepatic lesions in laying hens chronically exposed to rations containing different levels of aflatoxin B1. Arq Inst Biol, São Paulo, v.66, p.3943, 1999

PIER, A.C. Major biological consequences of aflatoxicosis in animal production. J Anim Sci, v.70, p.3964-3967, 1992.

RANDALL, C.J.; REECE, R.L. Colour atlas of avian histopathology. London : Mosby-Wolfe, 1996. 232p.

RIBEIRO, J.M.M. et al. Toxigenic mycoflora and mycotoxins (aflatoxins and ochratoxin A) in poultry feed in Rio de Janeiro, Brazil. In: INTERNATIONAL IUPAC SYMPOSIUM ON MYCOTOXINS AND PHYCOTOXINS, 2000, Guarujá. Abstract Book... Guarujá : International Union on Pure and Applied Chemistry, 2000. p.133.

SABINO, M. Variações de níveis de aflatoxina B1 em alimentos e rações animais no período de 1971 a 1979. Rev Inst Adolfo Lutz, v.40, p.153-158, 1980 .

SABINO, M. et al. Ocorrência de aflatoxina $\mathrm{B}_{1}$ em produtos alimentícios e rações animais, consumidos no Estado de São Paulo e em várias regiões do Brasil, no período de 1980 a 1987. Rev Inst Adolfo Lutz, v.48, p.81-85, 1988.

SANTURIO, J.M. et al. Aflatoxinas, ocratoxina A e zearalenona em grãos e rações destinadas ao consumo animal no sul do Brasil. In: ENCONTRO NACIONAL DE MICOTOXINAS, 1992, São Paulo. Anais... São Paulo : Instituto Adolfo Lutz, 1992. p.14.

SAS Institute. SAS ${ }^{\circledast}$ User's Guide: Statistics. Cary, NC : SAS Institute Inc., 1992. 1686p.

SAWHNEY, D.S.; VADEHRA, D.V.; BAKER, R.C. Aflatoxicosis in the laying japanese quail (Coturnix coturnix japonica). Poult Sci, v.52, p.465-473, 1973.

SCOTT, P.M. Natural poisons. In: HELRICH, K. Official methods of analysis of the Association of Official Analytical Chemists. 15. ed. Arlington : Association of Official Analytical Chemists (AOAC), 1990. v.2. p.1184-1213.

SOARES, L.M.V.; RODRIGUES-AMAYA, D.B. Survey of aflatoxins, ochratoxins A zearalenona and sterigmatocystin in some Brazilian foods by using multitoxin thin layer chromatografic method. J Assoc of Anal Chem, v.72, p.2226, 1989.

VASCONCELOS, A.C. Necropsia e remessa de material para laboratório em medicina veterinária. Brasília : ABEAS, 1988. 73p 